\title{
Heart valve mechanical prosthesis: The perfect match until it is not - A case report
}

\author{
IONELA MOVILEANU ${ }^{1}$, MIRUNA PEPA ${ }^{1}$, MARCELA CÂNDEA $^{2}$ and CORINA URECHE ${ }^{2}$ \\ ${ }^{1}$ First Cardiology Department, Emergency Institute of Cardiovascular Diseases and Transplant; \\ ${ }^{2}$ First Internal Medical Department, 'George Emil Palade’ University of Medicine, \\ Pharmacy, Sciences and Technology, 540136 Târgu Mureș, Romania
}

Received May 1, 2020; Accepted June 1, 2020

DOI: $10.3892 /$ etm.2020.8959

\begin{abstract}
Primary immune thrombocytopenia (ITP), formerly known as idiopathic thrombocytopenia, is defined by a reduced platelet count $<50 \times 10^{9}$ and is clinically manifested through signs and symptoms such as bleeding, ecchymosis and petechiae. Its coexistence with the implantation of the mechanical valves and the necessity of anticoagulation creates an additional risk of bleeding. Although mechanical valves are indispensable in prolonging and improving the quality of life in patients with valvular heart disease, in the context of an additional bleeding risk factor, their presence could represent a threat to life exposing the patient to major complications and leading to death. The purpose of this case report is to discuss the disadvantages and possible fatal complications of the association between mechanical valves and severe thrombocytopenia. A possible solution to these downsides could be found in the future search in regenerative medicine and tissue engineering of heart valves resulting in products that do not require anticoagulation and do not pose a threat to patients with thrombocytopenia.
\end{abstract}

\section{Introduction}

Usage of heart valve prostheses represented revolutionary treatment of valvular heart disease. Based on their nature, the valvular prostheses are classified in mechanical and bioprosthetic valves, each of them having inherent limitations.

Correspondence to: Dr Miruna Pepa, First Cardiology Department, Emergency Institute of Cardiovascular Diseases and Transplant, 50 Gheorghe Marinescu Street, 540136 Târgu Mureș, Romania

E-mail: pepamiruna@gmail.com

Abbreviations: ITP, primary immune thrombocytopenia; INR, international normalized ratio; CT, computer tomography

Key words: primary immune thrombocytopenia, mechanical heart valve, anticoagulation, vitamin $\mathrm{K}$ antagonists, bleeding, thrombotic events
Mechanical valves require permanent anticoagulation whereas biological ones are characterized by limited lifespan, requiring subsequent re-interventions (1). The choice of the prostheses is performed on the best match with patient's individualities. Although they offer a life quality improvement, their presence could represent a threat to life when other pathologies overlap. Thereby we are presenting the case of a male patient with aortic valve replacement with mechanical prosthesis implanted twenty years ago for post rheumatic severe stenosis of the aortic valve that is now diagnosed with a severe hematologic disease.

\section{Case report}

A 77-year male patient, carrier of a mechanical prosthesis (a 21-mm sized St. Jude bi-leaflet model) for post rheumatic severe stenosis of the aortic valve with effective oral anticoagulation and a prior history of hypertension and left bundle branch block, showed up at the emergency department with extensive and scattered ecchymosis on his body surface accompanied by petechial rash on the lower extremities, thorax and abdomen. Informed consent regarding the use of the patient medical records for educational purposes, excluding all personal identifiers, was signed by the patient during hospital admission. The patient denied bleeding, fever or headache and there was no history of drug overdose, alcohol intake or recreational drugs. On admission he was afebrile and normotensive. Abdominal and neurological examinations were normal. The heart rate at presentation was $60 \mathrm{bpm}$, with intermittent irregular rhythm. Laboratory examination revealed severe thrombocytopenia (platelet count 5,000/ $\mathrm{ll}$ ) and international normalized ratio (INR) 2.43 secondary to the administration of vitamin $\mathrm{K}$ antagonist anticoagulants. Transthoracic ultrasound revealed preserved cardiac cavities function with physiological dimensions. Evaluation of the valvular prosthesis showed mobile disks, free of attached masses and absence of suggesting imagining of pannus. In terms of function, the prosthesis presented good opening and complete closing with two small lateral regurgitation jets - characteristic for this prosthesis type and described in previous ultrasound examination. No para prosthetic leaks were identified. The pressure gradient across the prosthesis presented unchanged when compared with the previous examination and the indexed effective orifice area ruled out the patient-prosthesis mismatch. By joining the data resulting from 
the trans-thoracic ultrasound it was considered that the thrombocytopenia had no cardiac aetiology and further explorations in this direction were not performed. In the clinical context of isolated thrombocytopenia without a clinically apparent cause, a presumptive diagnosis of a haematological disorder was made. The peripheral smear showed normal appearing erythrocytes and neutrophil with a decreased number of platelets. Subsequently, the patient received intravenous steroids with a consecutive increase of platelet count to $48,000 / \mu 1$ then he was discharged home after initiation of oral treatment with steroids.

The patient was readmitted after 90 days from discharge, complaining of continuous headache accompanied by dizziness and confusion after a fall on the same level. The emergency computed tomography (CT) scan revealed a subdural hematoma in the right front-temporo-parietal region which did not require urgent surgical evacuation. The patient reported a constant level of the INR, in the therapeutic range, at weekly examinations. The emergency laboratory tests performed revealed a platelet count of $6,000 / \mu 1$ and efficient INR.

Considering the severe thrombocytopenia responsible for the increased risk of hematoma enlargement, the anticoagulation with vitamin $\mathrm{K}$ antagonists was delayed until spontaneous resolution was confirmed by several CT scans. Subsequently, intravenous steroid therapy and subcutaneous anticoagulation with twice a day Nadroparin 5700 UI were initiated. The following day the patient presented neurological status changes and the repeat tomography revealed a sudden growth in hematoma size (from 4 to $24 \mathrm{~mm}$ ) with compression and left midline shift. The decision to stop the anticoagulation therapy was taken and the drainage intervention was temporised in order try to correct the thrombocytopenia and to clear out the anticoagulant. Twelve hours later, the patient became anisocoric and unresponsive to verbal and painful stimuli, followed by cardio-respiratory arrest and unresponsive cardio-respiratory resuscitation. These were interpreted in the context of either a massive stroke embolized from the thrombosed mechanical valve or the presence of an active bleeding causing the hematoma extension.

\section{Discussion}

Based on the pathological mechanism thrombocytopenia can be classified as primary, when the low count of platelets is not associated with other systemic conditions, and secondary, when it is associated with infections, lymphoproliferative, autoimmune disorders or drug induced immunodeficiency. All these instances reflect the resulting effects of a decreased bone marrow platelet production, increased splenic sequestration or increased peripheral platelet destruction. Primary immune thrombocytopenia (ITP), formerly known as idiopathic thrombocytopenia (2), is defined by a reduced platelet count below $50 \times 10^{9}$ by a clinical picture of signs and symptoms such as bleeding, ecchymosis and petechiae. All the specified clinical manifestations amongst newly diagnosed ITP patients with a platelet count less than $50 \times 10^{9}$ purpura was present in $58 \%$ of cases, only $12 \%$ presented with bleeding while $28 \%$ were completely asymptomatic (3). Although it was thought to occur more often in early adulthood, a study found that the incidence of ITP is increasing with age with a peak among the population aged $>60$ years (4). Considering this information, when no other cytopenia are associated and the peripheral blood smear does not show other changes except the reduced number of thrombocytes, as in our patient, it is suggested that bone marrow examination is not mandatory in this group, as previously haematology guidelines indicated and not infrequently, the response to treatment is considered the best diagnostic test (5).

Even though several pathological mechanisms and diagnostic methods have become available, corticosteroids still remain the cornerstone for treating ITP in carriers of a mechanical aortic prosthesis and oral anticoagulation, despite other first line treatments such as immunoglobulins G or Rho (D). This is due to possible severe adverse events as thrombosis or accelerated haemolysis, increased plasma viscosity after immunoglobulin $\mathrm{G}$ administration, or RBC antigen-antibody-mediated complement activation after anti-D treatment, contributing most. Regarding splenectomy as second line treatment, despite the high success rate and the rapid response, in patients with comorbidities, sometimes it does not represent a suitable option (5).

Extensive research of the scientific literature revealed limited information regarding cases of patients with heart valve prostheses and associated thrombocytopenia. The few existing reports presented the appearance within the immediate time span after the surgical or interventional procedure (6-8). Related to the thrombocytopenia causes, studies present numerous and varied conditions that can trigger it. In the clinical scenario of patients requiring permanent anticoagulation the most frequent etiology is heparin-induced thrombocytopenia (9). Unfractionated heparin causes a decrease in the platelet number by its additional binding to platelets and endothelial cells besides antithrombin. Thrombocytopenia appears subsequently to the antibody activation induced by heparin, directed against the bond between platelet factor 4 and unfractionated heparin (10), causing an antibody-mediated syndrome. This was not the case of our patient because he was anticoagulated with vitamin $\mathrm{K}$ antagonists.

The cardiac aetiology of thrombocytopenia is related to the presence of a mechanical valve causing mechanical destruction. This scenario could be secondary only to usage of prosthetic valves with poor hemodynamic that create major trans-prosthetic gradients such the models Starr Edward - ball in cage. The patient presented in the case had a St. Jude bi-leaflet mechanical valve, the most commonly used, known to have improved hemodynamic performance (11).

The hematoma formed and extended due to the platelet low count and the anticoagulant state of the patient. Absence of anticoagulation in patients with St. Jude heart valve prostheses is reported to be associated with thrombosis of the valve or embolism in a proportion of $12 \%$ for the aortic position (12). With the presence of the hematoma we opted for the switch of the vitamin $\mathrm{K}$ antagonist toward the lower dose of low-molecular weight heparin to obtain a well-controlled anticoagulation and avoid INR fluctuations.

The existing heart valve substitutes are imperfect, their usage and the complementary therapy required to be associated could expose the patient to major complications and can lead to death. A solution to these downsides could be found in the future research in regenerative medicine and tissue engineering of heart valves. At present, studies are in different levels of the research: in laboratory (13-15), implanting in animal models $(16,17)$ and even translational to the surgical clinics $(18)$. 
In conclusion, mechanical heart valve substitutes offer an improved quality of life in patients with valvular heart disease, however, with their inherent downsides - the need of permanent anticoagulation, that primarily determine the major potential complications such as bleeding or thrombotic events. When another pathology overlaps, such as autoimmune thrombocytopenia, the management of these patients can often be difficult, as the dynamic balance between activated procoagulants and endogenous anticoagulants is impaired. This case demonstrates that thrombocytopenia may be present as a separate pathological entity in patients with mechanical valves contributing to the risk of bleeding and death of patients.

\section{Acknowledgements}

Not applicable.

\section{Funding}

No funding was received.

\section{Availability of data and materials}

The datasets used and/or analyzed during the current study are available from the corresponding author on reasonable request.

\section{Authors' contributions}

MC and CU treated the patient, were involved in the conception and design of the study, contributed in the analysis and interpretation of the data, and revised the manuscript. IM and MP acquired the patient data, were also involved in the analysis and interpretation of the data and drafted the manuscript. All authors read and approved the final version of the manuscript and agree to be accountable for all aspects of the study in ensuring that questions related to the accuracy or integrity of any part of the study are appropriately investigated and resolved.

\section{Ethics approval and consent to participate}

Not applicable.

\section{Patient consent for publication}

Informed consent regarding the use of the patient medical records for educational purposes, excluding all personal identifiers, was signed by the patient during hospital admission.

\section{Competing interests}

The authors declare that they have no competing interests.

\section{References}

1. Vangeldorp M, Jamieson WRE, Kappetein AP, Ye J, Fradet GJ, Eijkemans MJC, Grunkemeier GL, Bogers JJC and Takkenberg JJM: Patient outcome after aortic valve replacement with a mechanical or biological prosthesis: Weighing lifetime anticoagulant-related event risk against reoperation risk. J Thorac Cardiovasc 37: 881-886, 2009.
2. Rodeghiero F, Stasi R, Gernsheimer T, Michel M, Provan D, Arnold DM, Bussel JB, Cines DB, Chong BH, Cooper N, et al: Standardization of terminology, definitions and outcome criteria in immune thrombocytopenic purpura of adults and children: Report from an international working group. Blood 113: 2386-2393, 2009.

3. Neylon AJ, Saunders PW, Howard MR, Proctor SJ and Taylor PR; Northern Region Haematology Group: Clinically significant newly presenting autoimmune thrombocytopenic purpura in adults: A prospective study of a population-based cohort of 245 patients. Br J Haematol 122: 966-974, 2003.

4. Abrahamson PE, Hall SA, Feudjo-Tepie M, Mitrani-Gold FS and Logie J: The incidence of idiopathic thrombocytopenic purpura among adults: A population-based study and literature review. Eur J Haematol 83: 83-89, 2009.

5. McCrae K: Immune thrombocytopenia: No longer 'idiopathic'. Cleve Clin J Med 78: 358-373, 2011.

6. Piccardo A, Rusinaru D, Petitprez B, Marticho P, Vaida I, Tribouilloy $\mathrm{C}$ and Caus T: Thrombocytopenia after aortic valve replacement with freedom solo bioprosthesis: A propensity study. Ann Thorac Surg 89: 1425-1430, 2010.

7. Mujtaba SS, Ledingham S, Shah AR, Schueler S, Clark S and Pillay T: Thrombocytopenia after aortic valve replacement: Comparison between sutureless perceval S Valve and Perimount Magna Ease Bioprosthesis. Braz J Cardiovasc Surg 33: 169-175, 2018.

8. Kizilay M, Elbir F, Aglar AA, Vural U, Balci AY and Yekeler İ: An overlooked fact: Thrombocytopenia following bioprosthetic aortic valve replacement. Kardiochir Torakochirurgia Pol 16: 19-26, 2019 (In Polish).

9. Corbett TL, Elher KS and Garwood CL: Successful use of fondaparinux in a patient with a mechanical heart valve replacement and a history of heparin-induced thrombocytopenia. J Thromb Thrombolysis 30: 375-377, 2010.

10. Warkentin TE, Greinacher A, Koster A and Lincoff AM: Treatment and prevention of heparin-induced thrombocytopenia. American College of Chest Physicians Evidence-Based Clinical Practice Guidelines (8th Edition). Chest 133: S340-S380, 2008.

11. Emery RW, Krogh CC, Arom KV, Emery AM, Benyo-Albrecht K, Joyce LD and Nicoloff DM: The St. Jude Medical cardiac valve prosthesis: A 25-year experience with single valve replacement. Ann Thorac Surg 79: 776-782, discussion 782-783, 2005.

12. Salem DN, O'Gara PT, Madias C and Pauker SG: Valvular and structural heart disease: American College of Chest Physicians Evidence-Based Clinical Practice Guidelines (8th edition). Chest 133: S593-S629, 2008

13. Tardalkar K, Desai S, Adnaik A, Bohara R and Joshi M: Novel approach toward the generation of tissue engineered heart valve by using combination of antioxidant and detergent: A potential therapy in cardiovascular tissue engineering. Tissue Eng Regen Med 14: 755-762, 2017.

14. Zhang BL, Bianco RW and Schoen FJ: Preclinical assessment of cardiac valve substitutes: Current status and considerations for engineered tissue heart valves. Front Cardiovasc Med 6: 72, 2019.

15. Movileanu I, Brinzaniuc K, Harpa M, Nistor D, Cotoi O, Preda T, Al Hussein H, Moldovan O, Man A, Harceaga L, et al: Pressurized perfusion system for obtaining completely acellular pulmonary valve scaffolds for tissue engineering. ARS Med Tomitana 25: 149-156, 2019.

16. Harpa M, Movileanu I, Sierad L, Cotoi O, Suciu H, Preda T, Nistor D, Sircuța C, Brânzaniuc K, Deac R, et al: In vivo testing of xenogeneic acellular aortic valves seeded with stem cells. Rev Rom Med Lab 24: 343-346, 2016.

17. Reimer J, Syedain Z, Haynie B, Lahti M, Berry J and Tranquillo R: Implantation of a tissue-engineered tubular heart valve in growing lambs. Ann Biomed Eng 45: 439-451, 2017.

18. Boethig D, Horke A, Hazekamp M, Meyns B, Rega F, Van Puyvelde J, Hübler M, Schmiady M, Ciubotaru A, Stellin G, et al: A European study on decellularized homografts for pulmonary valve replacement: Initial results from the prospective ESPOIR Trial and ESPOIR Registry data. Eur J Cardio-Thorac Surg 56: 503-509, 2019. 